\title{
Análise do movimento de um ponto de luz sobre um plano inclinado
}

\author{
Motion analysis of a light spot on inclined plane \\ Priscila Freitas-Lemes ${ }^{* 1,2}$, Douglas Carlos Vilela 10 , Murilo Gelly Guarnieri ${ }^{1}$, Rafael de Oliveira
Prado $^{1}$, Thiago Filipe de Medeiros ${ }^{1}$, José Silvério Edmundo Germano $^{1}$ \\ ${ }^{1}$ Instituto Tecnológico de Aeronáutica, São José dos Campos, SP, Brasil \\ ${ }^{2}$ Universidade do Vale do Paraíba, São José dos Campos, São Paulo, Brasil
}

Recebido em 28 de Março, 2018. Revisado em 13 de Julho, 2018. Aceito em 01 de Agosto, 2018.

\begin{abstract}
Estudos recentes na área do ensino de engenharia têm mostrado a importância de se desenvolver novas abordagens nas aulas de laboratório experimentais de física nas séries iniciais dos cursos de engenharia. Neste artigo apresentamos os resultados de uma proposta de desenvolvimento, baseada na metodologia PBL, de um experimento de fácil construção e montagem, porém de análise e interpretação dos dados bastante complexos. O experimento denominado, pêndulo a laser, tem o objetivo de analisar o movimento de um ponto de luz produzido por um laser que está acoplado num pêndulo de haste rígida cuja oscilação do ponto de luz ocorre sobre um plano inclinado de ângulo variável. Os dados experimentais foram obtidos por meio das filmagens com o uso do software de rastreamento Tracker®enquanto as análises dos modelos teóricos desse problema, bem como os gráficos e as curvas ajustadas foram obtidos com o uso do software de análise de dados GNU Octave®. Por fim, uma simulação em Python de todo o experimento foi construída a fim de auxiliar no processo de visualização e entendimento do problema analisado.
\end{abstract}

Palavras-chave: Pêndulo físico, laser, plano inclinado, Python.

Recent studies in engineering education have shown the importance of developing new approaches in experimental physics lab in the initial series of engineering courses. In this paper it was analyzed the motion of a light spot produced by an oscillating laser on a rigid rod pendulum over a inclined plane. The main parameter in this paper is the distance between the light spot and the pendulum rotating axis. The study of this parameter was made by the analysis of its behavior with respect to time, which was accomplished by comparison of data obtained by two different methods: the analysis of filming of the experiment and the theoretical prevision aligned with the curves fitting over the experimental data. The empirical data were obtained from the video analysis using the Tracker ${ }^{\circledR}$ software and the graphics and fitting curves were obtained with use of the data analysis software GNU Octave ${ }^{\circledR}$. This experiment is simple and interesting as well as can be done with accessible resources. Finally, a Python simulation of the whole experiment was constructed in order to assist in the process of visualization and understanding of the analyzed problem.

Keywords: Physical Pendulum, laser, inclined plane, Python.

\section{Introdução}

No século XXI a tecnologia avançou produzindo um conjunto de modificações no cotidiano das pessoas, nas profissões e no segmento educacional, com impacto desde as crianças até os adultos [1]. Entretanto, o desenvolvimento tecnológico e a inovação são assuntos pouco debatidos entre estudantes de engenharia. Esse debate precisa ser estimulado no ambiente acadêmico, pois a inovação é um elemento importante para a transformação de conhecimento em riqueza, principalmente em países com acentuada desigualdade social. A interação entre instituições de ensino e pesquisa, empresas e governo é salutar para a solução desse problema complexo [2].

*Endereço de correspondência: piprisc@gmail.com
O laboratório de física numa escola de engenharia, tem um papel fundamental na formação científica dos estudantes, pois os fenômenos descritos por leis e teorias que permeiam a ciência, na sua maioria, tem que ter de alguma forma uma comprovação experimental afim do modelo teórico analisado ter algum fundamento.

Discutir o papel e a importância de se aprimorar os experimentos de física vem sendo estudado por alguns pesquisadores ([3]; [4]; [5]).

Borges [4] ressalta a importância do laboratório no ensino de ciências, onde os cientistas utilizam métodos, mas isso não significa que haja um método científico que determine exatamente como fazer para produzir conhecimento. O laboratório pode proporcionar excelentes oportunidades para que os estudantes testem suas pró- 
prias hipóteses sobre fenômenos particulares, para que planejem suas ações, e as executem, de forma a produzir resultados dignos de confiança.

É preciso destacar que no curso de engenharia, os estudantes devem estar em contato direto com o desenvolvimento de situações práticas. E para solucionar essas situações práticas é cada vez mais necessário o conhecimento das Tecnologias Digitais de Informação e Comunicação (TDIC) recentes, como apoio para auxiliar na solução desses problemas e o ambiente do laboratório de física é o local para que os estudantes entrem em contato com essas tecnologias.

Desta forma, Pekelman e Mello-Jr [6] destacam a relação entre o laboratório e o processo de simulação e modelagem.

Se o engenheiro deve ser capaz de aplicar a ciência e a tecnologia, a adaptação dos conhecimentos científicos e tecnológicos às necessidades humanas, nada mais justo, que no seu período de ensino no curso de Engenharia, lhe seja proporcionado a possibilidade de se deparar e manipular as principais tecnologias básicas em condições próximas, simuladas, às do mundo real, ou melhor, profissional. Podemos dizer, que os laboratórios em uma instituição de ensino de Engenharia modelam situações reais (modelo - simplificação da realidade) e utilizam estes modelos em simulações.

Para a análise desse problema e compreensão do efeito das diversas variáveis que aparecem na resolução desse problema, uma simulação deste projeto foi feita utilizando o software VPython com a versão 2.7.10 da linguagem Python. A simulação está disponível em: http://161. 24.5.141/laser/

A proposta teve como metodologia o Problem Based Learning - PBL. O PBL vem sendo utilizado em diversas atividades de laboratórios de física para ensino superior com relativo êxito $[7 ; 8]$.

Bouquet [8] destaca que existem vários parâmetros que reduzem o apelo de uma abordagem baseada em projetos (PBL) em currículos de física na universidade. Afinal, geralmente requer um grande conjunto de versatilidade e muitas vezes equipamento caro, requer mais tempo do que os tradicionais ensinamentos, e para os professores isso pode ser desestabilizador.

Pensando na temática do pêndulo, esse artigo propõe a construção de um sistema acoplado de um pêndulo físico com um laser e o movimento o ponto de luz ocorre sobre um plano inclinado ilustrado na Figura 1. Neste experimento é usada a temática do Movimento Harmônico Simples, movimento oscilatório ocorrido quando a aceleração e a força resultante são proporcionais e opostas ao deslocamento [3]. Aqui o MHS será aplicado ao pêndulo físico, suspenso de modo a oscilar livremente em um plano vertical, em torno de um eixo passando pelo corpo [12]. Este tipo de situação existe em mapeamento através de presente em sensores de movimento RGB como o existente no Kinect[13]. A reconstrução de objetos é realizada através de modelagem matemá-

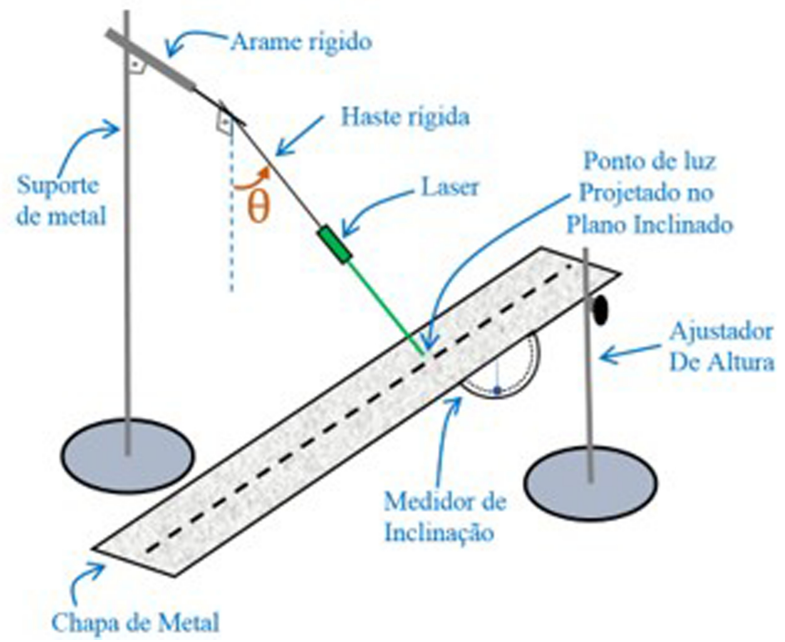

Figura 1: Esquema da montagem do experimento.

tica. Deste modo, ter contato com um experimento que permite adequar um laser pode proporcionar ao aluno a capacidade de modelar um sistema mais complexo como kinect doravante.

Relatamos o resultado de uma atividade pensada e desenvolvida por estudantes do curso de engenharia do Instituto Tecnológico de Aeronáutica (ITA) ao longo do curso de Física Experimental I na perspectiva do PBL.

O presente artigo está dividido da seguinte forma: na seção 2 apresentamos resumidamente os fenômenos físicos, os detalhes teóricos da montagem e funcionamento do sistema. Na seção 3 exibimos o sistema em uso no laboratório. Na seção 4 apresentamos os resultados obtidos nas diferentes configurações experimentais. Na seção 5 apresentamos a simulação em Python desenvolvida exclusivamente para esse sistema. E, por fim, na seção 6 apresentamos as conclusões.

\section{O pêndulo físico com laser acoplado ao plano inclinado: fundamentação teórica.}

Veja um esquema da montagem do experimento na Figura 1. O movimento de um ponto de luz produzido pela oscilação de um laser preso a um pêndulo de haste rígida sobre um plano inclinado pode ser analisado tomando a composição de dois sistemas mais simples: pêndulo físico e configuração espacial dos equipamentos envolvidos.

O pêndulo físico fornece a relação entre ângulo de abertura do pêndulo $(\theta)$ e tempo $(t)$, enquanto a configuração espacial fornece a relação entre a distância do ponto de luz ao eixo de rotação do pêndulo $(\mathrm{R})$ e o ângulo $\theta(\mathrm{t})$. Dessa forma a análise do movimento do ponto de luz pode ser feita unindo-se os dois sistemas que fornecerão, por meio da composição, R(t). 


\subsection{Pêndulo físico sob a ação de uma força resistiva proporcional à velocidade angular}

Na Figura 2 é apresentado o esquema de um pêndulo físico sob a ação de uma força resistiva proporcional à velocidade angular.

Onde $\theta$ é ao ângulo de abertura do pêndulo com relação à vertical, $R_{\mathrm{CM}}$ é a distância do eixo de rotação do pêndulo (ponto $\mathrm{O}$ ) até o centro de massa da haste que oscila, $\mathrm{F}_{\mathrm{A}}$ é a força resistiva, $\mathrm{P}$ é o peso da haste e do laser e g é a aceleração da gravidade (veja Figura 2).

Pela teoria de movimento rotacional [9] temos que o torque aplicado no corpo oscilante pode ser calculado pelas equações (1) e (2) abaixo.

$$
\begin{gathered}
\vec{\tau}_{0}=\vec{R}_{C M} x\left(\vec{P}_{\theta}+\vec{F}_{A}\right) \\
\vec{\tau}_{0}=\left(R_{C M} \hat{u}_{R}\right) x\left(-P \operatorname{sen} \theta \hat{u}_{\theta}-k \omega \hat{u}_{\theta}\right) \\
\vec{\tau}_{0}=-R_{C M}(P \operatorname{sen} \theta+k \omega)\left(\hat{u}_{R} x \hat{u}_{\theta}\right) \\
\vec{\tau}_{0}=-R_{C M}\left(P \operatorname{sen} \theta+k \frac{d \theta}{d t}\right) \hat{u}_{z} \\
\vec{\tau}_{0}=I_{0} \vec{\alpha}=I_{0} \alpha \hat{u}_{z}=I_{0} \frac{d^{2} \theta}{d t^{2}} \hat{u}_{z}
\end{gathered}
$$

Portanto:

$$
\begin{gathered}
-R_{C M}\left(P \operatorname{sen} \theta+k \frac{d \theta}{d t}\right)=I_{0} \frac{d^{2} \theta}{d t^{2}} \\
\frac{d^{2} \theta}{d t^{2}}+\frac{R_{C M} k}{I_{0}} \frac{d \theta}{d t}+\frac{R_{C M} m g}{I_{0}} \operatorname{sen} \theta=0
\end{gathered}
$$

Em forma compacta tem-se:

$$
\begin{gathered}
\gamma=\frac{R_{C M} k}{I_{0}} \\
\zeta=\frac{R_{C M} m g}{I_{0}} \\
\frac{d^{2} \theta}{d t^{2}}+\gamma \frac{d \theta}{d t}+\zeta \operatorname{sen} \theta=0
\end{gathered}
$$

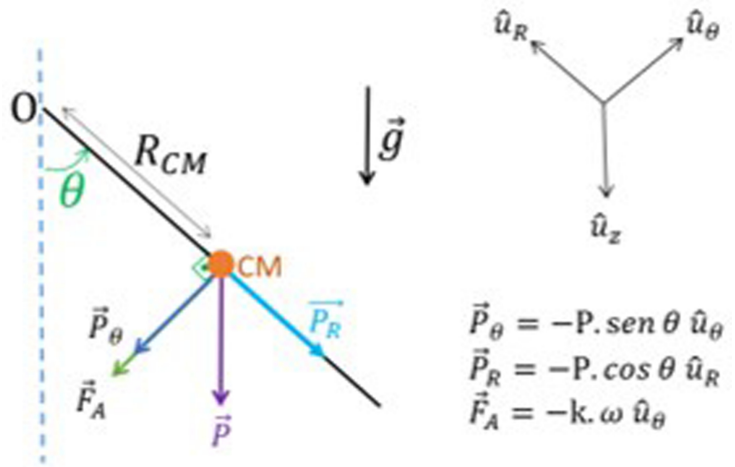

Figura 2: Esquema de pêndulo sob a ação de uma força resistiva proporcional à velocidade angular com os vetores ûr (relativo ao comprimento do pêndulo), û $\theta$ 9relativo ao ângulo do pêndulo) e ûz (relativo à eixo z da coordenada cartesiana).
A equação diferencial ordinária (6) não apresenta solução analítica, dessa forma outras abordagens devem ser utilizadas a fim de se obter uma relação $\theta(\mathrm{t})$.

Pode-se utilizar a abordagem numérica para a determinação da solução, assim como realizar simplificações a fim de se obter uma EDO que possui solução analítica.

\subsection{Resolução simplificada do movimento do pêndulo físico sob a ação de uma força resistiva proporcional à velocidade angular}

A simplificação aplicada à EDO (6) consiste em tomar $\operatorname{sen} \theta$ aproximadamente igual a $\theta$. Esta aproximação mostra-se adequada para casos em que $\theta$ é pequeno, porém não tão boa à medida que $\theta$ torna-se maior.

$$
\operatorname{sen} \theta \approx \theta
$$

Substituindo a expressão (7) em (6), tem-se:

$$
\frac{d^{2} \theta}{d t^{2}}+\gamma \frac{d \theta}{d t}+\zeta \theta \approx 0
$$

Com a teoria matemática acerca de equações diferenciais ordinárias [9], é possível chegar a uma solução analítica para a EDO 8). Dependendo da relação entre $\gamma$ e $\zeta$ existem certos conjuntos de solções que podem ser analisadas. No caso estudado, que se remete a um movimento oscilatório subcrítico na situação de $\gamma<\zeta$, temos que a solução é dada pela equação (9).

$$
\theta(t)=e^{r_{r} t}\left[c_{1} \cos \left(r_{i} t\right)+c_{2} \operatorname{sen}\left(r_{i} t\right)\right]
$$

$$
\begin{gathered}
r_{i}=\sqrt{\zeta-\frac{\gamma^{2}}{4}} \\
r_{r}=-\frac{\gamma}{2}
\end{gathered}
$$

\subsubsection{Vínculo geométrico da montagem do experimento}

Partindo-se do princípio que a relação entre $\theta$ e tempo já é conhecida, seja pela solução simplificada ou exata da EDO (6), pode-se analisar a geometria da montagem do experimento e assim determinar uma expressão que relacione um parâmetro de interesse ao ângulo $\theta(\mathrm{t})$. O esquema da montagem do experimento pode ser consultado na Figura 3.

Onde $\alpha$ é o ângulo de inclinação do plano inclinado em relação à horizontal, $\mathrm{R}$ é a distância entre o ponto de luz projetado no plano inclinado e o eixo de rotação do pêndulo, B é a distância entre o eixo de rotação do pêndulo e o plano inclinado seguindo um segmento de reta vertical. Tem-se pela geometria da montagem:

$$
\begin{gathered}
B=R \cos (\theta)+R \operatorname{sen}(\theta) \tan (\alpha) \\
B=R \frac{\cos (\theta) \cos (\alpha)+\operatorname{sen}(\theta) \operatorname{sen} \theta(\alpha)}{\cos (\alpha)}
\end{gathered}
$$

Revista Brasileira de Ensino de Física, vol. 41, nº 1, e20180090, 2019 


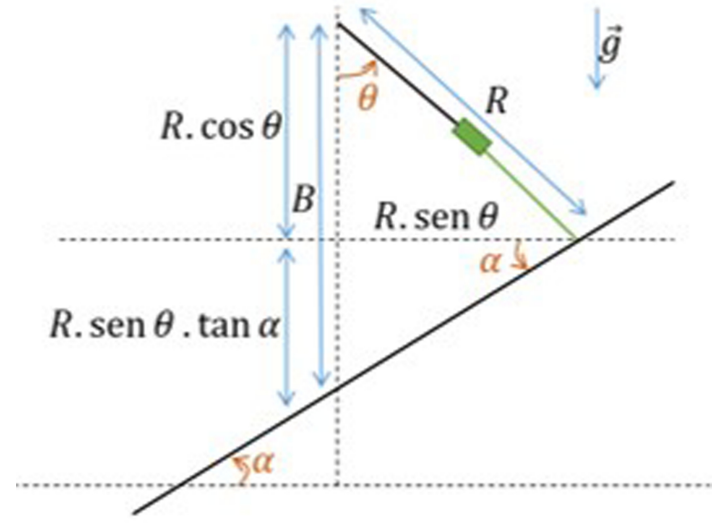

Figura 3: Esquema da montagem do experimento.

$$
\begin{gathered}
B=R \frac{\cos (\theta-\alpha)}{\cos \theta(\alpha)} \\
R=B \cos (\alpha) \sec (\theta-\alpha)
\end{gathered}
$$

A equação (10) é válida em todo instante de tempo, e como $\theta$ varia com o tempo, $\mathrm{R}$ também variará. A relação entre R e t está explicitada na equação (11).

$$
R(t)=B \cos (\alpha) \sec (\theta(t)-\alpha)
$$

Com o conhecimento de $\mathrm{R}$ em $\mathrm{t}$ igual a zero e $\theta$ em $\mathrm{t}$ igual à zero pode-se eliminar $\mathrm{B}$ da equação:

$$
R(0)=B \cos (\alpha) \sec (\theta(0)-\alpha)
$$

Com as equações 111 e 12 tem-se:

$$
\begin{gathered}
R(t)=\frac{R_{0}}{\sec \left(\theta_{0}-\alpha\right)} \sec (\theta(t)-\alpha) \\
R(t)=R_{0} \frac{\cos \left(\theta_{0}-\alpha\right)}{\cos (\theta(t)-\alpha)}
\end{gathered}
$$

Uma das grandezas principais analisadas nesse trabalho é a distância do ponto projetado no plano até o eixo de rotação onde o pêndulo se encontra preso em função do tempo, ou seja, $\mathrm{R}$ em função de $\mathrm{t}(\mathrm{R}(\mathrm{t}))$. Para a determinação de $\mathrm{R}(\mathrm{t})$ bastam as equações (6) e (9).

Por fim, após a fundamentação teórica percebemos que a determinação de $\mathrm{R}_{0}, \theta_{0}, \alpha, \gamma$ e $\zeta$ é vital para a análise do movimento. Apesar de alguns parâmetros serem facilmente determinados - como $\alpha$, que é obtido consultando-se o medidor de inclinação preso ao plano inclinado, $\mathrm{R}_{0}$ e $\theta_{0}$, que são obtidos com o uso do software de análise de vídeo, outros parâmetros são de trabalhosa obtenção matemática, como $\gamma$ e $\zeta$

Para a determinação dos parâmetros $\gamma$ e $\zeta$ podem ser utilizados étodos numéricos de ajuste de curva aplicados aos dados obtidos experimentalmente. Nestes casos, $\gamma$ e $\zeta$ são determinados para que a solução teórica possua um perfil similar ao da curva experimental.

\section{Materiais e métodos}

Uma montagem experimental do sistema proposto foi montada. Utilizou-se para a montagem do experimento:

- 1 Suporte de metal ( $\approx$ 1,20 m de altura);

- 1 Laser (Potência: 5mw. Comprimento de onda: $532 \mathrm{~nm})$;

- 1 Haste de metal ( $\approx 30 \mathrm{~cm}$ de comprimento) com furo passante;

- 1 Chapa metálica - Plano Inclinado $(\approx 1,20 \mathrm{~m}$ de comprimento);

- 1 Ajustador de altura para a chapa;

- 1 Smartphone com resolução mínima de 240 frames/s;

- 1 Suporte de smartphone;

- Computador com o GNU Octave®[10] e Tracker ${ }^{\circledR}[11]$.

Na montagem experimental o laser foi preso à extremidade da haste de metal, o arame rígido foi preso à extremidade do suporte de metal, o medidor de inclinação foi encaixado à chapa metálica e a haste foi encaixada ao suporte de metal por meio do arame que passou por seu furo.

Posteriormente, o plano inclinado foi montado unindose a chapa metálica ao ajustador de altura, e a altura foi configurada de modo a se obter um ângulo de inclinação de 10 graus. O suporte de metal foi posicionado de modo que a haste oscilasse num plano perpendicular ao plano definido pela chapa de metal.

Após isso, o botão de liga/desliga do laser foi fixo para que a emissão não cessasse durante o experimento. A montagem física do experimento pode ser consultada na Figura 4.

Para a posterior realização da filmagem, o smartphone foi fixo em seu suporte e ambos foram posicionados de modo a se obter uma filmagem adequada para a futura análise de vídeo, observe a linha de visada da câmera da Figura 5. A escolha do smartphone se deve ao fato de que ele possui uma cadência de 240 frames por segundo

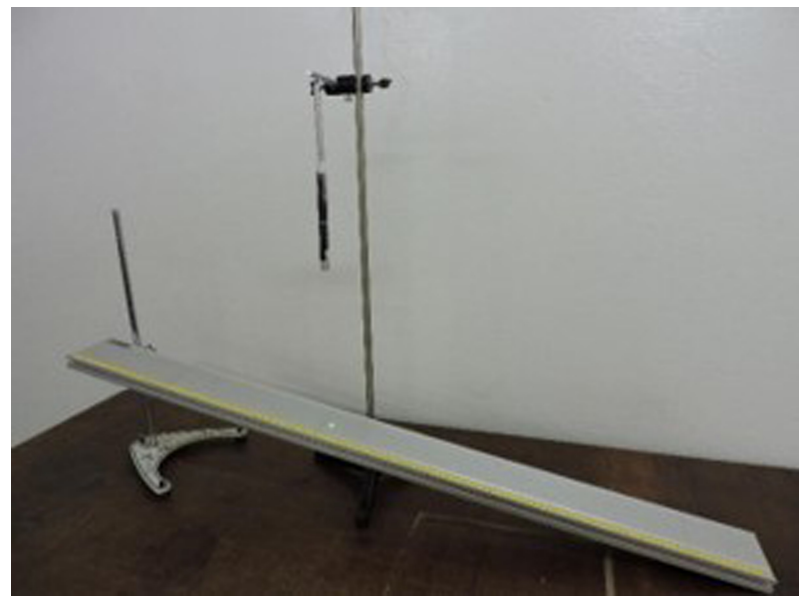

Figura 4: Foto da montagem física do experimento. 


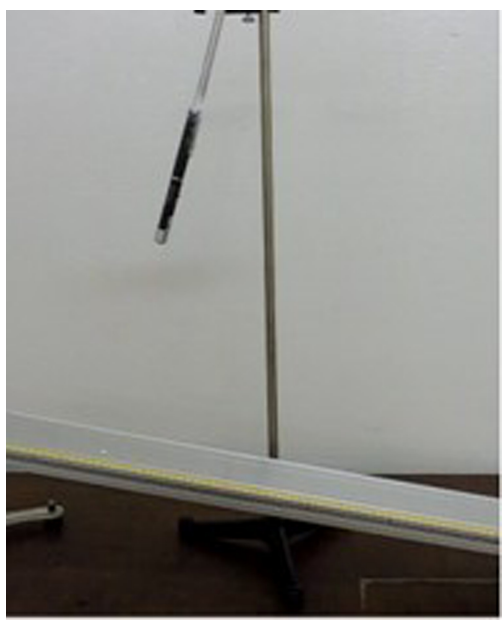

Figura 5: Foto do sistema haste-laser oscilando.

e dessa forma proporciona um maior números de dados para se analisar, além de um rastreamento mais confiável pelo software de análise de vídeo.

\subsection{Descrição da execução do experimento}

$\mathrm{Na}$ execução desse trabalho, diferentes inclinações do plano inclinado $\alpha$ e do ângulo de abertura do pêndulo $\theta$ (ângulo inicial) foram examinadas. Observe na Tabela 1 as configurações angulares que foram adotadas. $\mathrm{O}$ experimento em execução pode ser visualizado na Figura 5.

Para a análise dos vídeos, foi utilizado o software de rastreamento Tracker®, que é um software livre de análise de vídeo e modelagem de experimentos [11].

Para a produção de gráficos, ajuste de curvas, e manipulação matemática dos dados foi utilizado o software GNU Octave ${ }^{\circledR}$. Esse é um software livre destinado principalmente a cálculos numéricos com extensas capacidades gráficas para visualização e manipulação de dados [10].

\section{Resultados}

Utilizou-se para o modelamento teórico da oscilação do pêndulo a solução simplificada descrita pela EDO (8), pois: a amplitude angular $\left(\theta_{0}\right)$ se manteve sempre menor que 30 graus durante o experimento. Ângulos $\theta_{\mathrm{o}}$ maiores faziam com que o ponto de luz saísse do plano inclinado. As filmagens utilizadas possuíam curto tempo

Tabela 1: Diferentes configurações angulares utilizada na execução do experimento.

\begin{tabular}{ll}
\hline & Configuração utilizada \\
\hline Configuração 1 & $\alpha=1^{\circ}$ e $\theta<15^{\circ}$ \\
Configuração 2 & $\alpha=1^{\circ}$ e $\theta>15^{\circ}$ \\
Configuração 3 & $\alpha=2^{\circ}$ e $\theta<15^{\circ}$ \\
Configuração 4 & $\alpha=2^{\circ}$ e $\theta>15^{\circ}$ \\
\hline
\end{tabular}

de duração (dessa forma o efeito de defasagem causado pela simplificação da EDO não se torna perceptível).

A equação (9) serviu como função base para a realização do ajuste de curvas, pois após a realização do experimento, notou-se que os dados experimentais possuíam um perfil muito próximo da solução descrita pela equação 9 .

Para a realização do ajuste de curvas, primeiramente determinou-se as exponenciais moduladoras, utilizando os pontos de máximo e mínimo locais aliados à regressão linear nos dados de $\theta$ e tempo numa tabela log-log, em seguida determinou-se a velocidade angular média, baseado no tempo entre cada pico de máximo e mínimo, e finalmente determinou-se as constantes $c_{1}$ e $c_{2}$ utilizando-se os dados de um ponto próximo da posição de equilíbrio.

\subsection{Configuração experimental 1}

As análises sobre a configuração experimental 1 podem ser consultados nas Figuras 6, 7 e 8. Na Figura 6 é apresentada a comparação entre os dados experimentais e a solução teórica ajustada baseada na relação entre o ângulo de abertura do pêndulo $\theta$ e o tempo decorrido t sobre o plano inclinado em $10^{\circ}$ e com amplitude pequena de $\theta$. A curva resultante do ajuste de curvas possui os coeficientes explicitados na Tabela 2.

Os coeficientes $\gamma, \zeta$, assim como a relção $\mathrm{k} / \mathrm{mg}$ podem ser determinados a partir de $r_{i}$ e $r_{r}$, conforme as equações (4), (5), 15) e 16.

$$
\gamma=-2 r_{r}
$$

Tabela 2: Coeficientes da equação (9) que define a curva verde explicitada na Figura 6.

\begin{tabular}{llll}
\hline $\mathrm{c}_{1}\left(\mathrm{rad}^{-1}\right)$ & $\mathrm{c}_{2}\left(\mathrm{rad}^{-1}\right)$ & $\mathrm{r}_{\mathrm{i}}\left(\mathrm{s}^{-1}\right)$ & $\mathrm{r}_{\mathrm{r}}\left(\mathrm{s}^{-1}\right)$ \\
\hline$-0,16576$ & 0,10704 & 0,05669 & 9,11487 \\
\hline
\end{tabular}

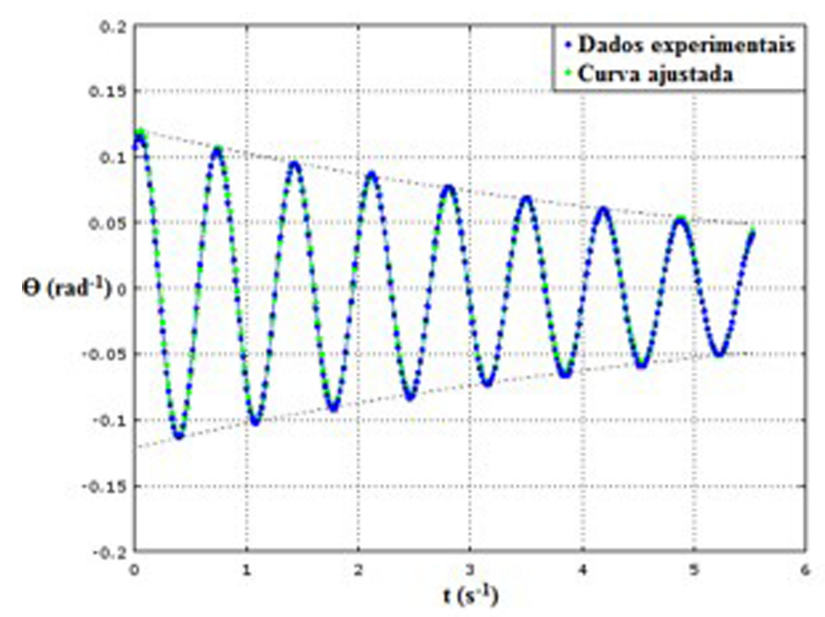

Figura 6: Gráfico com a comparação entre os dados experimentais (curva azul) e a solução teórica ajustada (curva verde) baseada na relação entre o ângulo de abertura do pêndulo $(\theta)$ e o tempo decorrido $(\mathrm{t})$. 


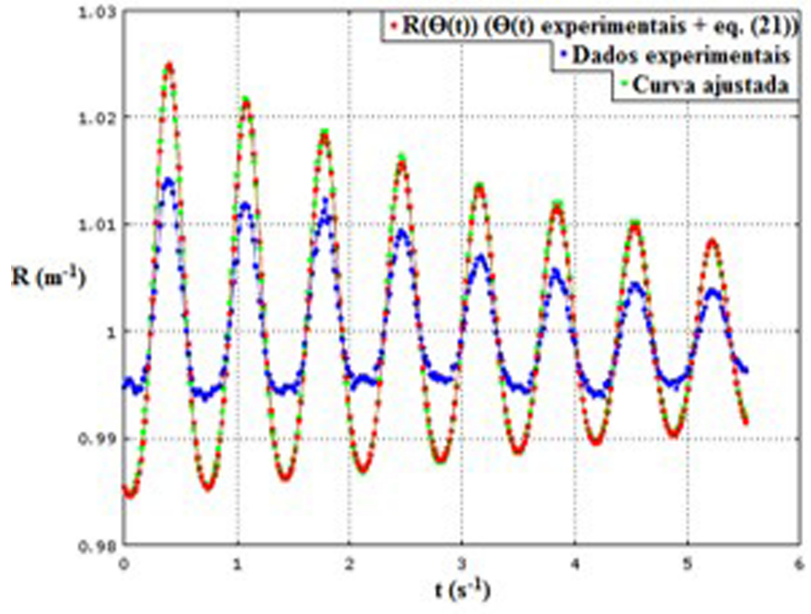

Figura 7: Gráfico com a comparação entre os dados experimentais, a solução teórica ajustada e a relação composta entre $\mathrm{R}(\theta(\mathrm{t}))$ para $\theta$ experimentais.

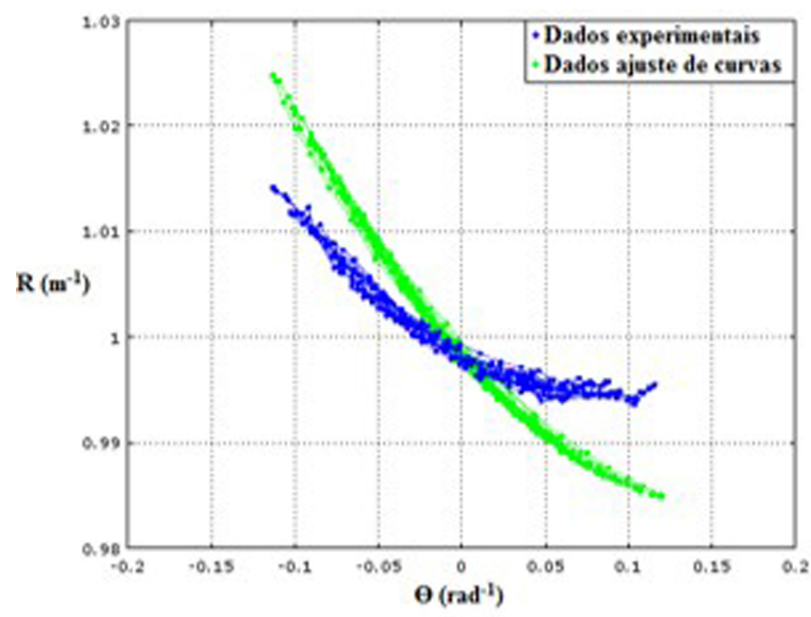

Figura 8: Gráfico com a comparação entre os dados experimentais (curva azul) e a solução teórica ajustada (curva verde) baseada na relação entre a distância do ponto de luz até o eixo de rotação do pêndulo $(R)$ e o ângulo de abertura do pêndulo $(\theta)$.

Tabela 3: Coeficientes $\gamma$, $\zeta$, e a relação $\mathrm{k} / \mathrm{mg}$ para o experimento para configuração experimental 1 .

\begin{tabular}{lll}
$\gamma\left[\left(\mathrm{s}^{-1}\right)^{-1}\right]$ & $\zeta\left[\left(\mathrm{s}^{-2}\right)^{-1}\right]$ & $\mathrm{k} / \mathrm{mg}\left[\mathrm{s}^{-1}\right]$ \\
\hline 0,33153 & 83,108 & 0,0039891
\end{tabular}

$$
\zeta=r_{i}^{2}+r_{r}^{2}
$$

Com as equações (4), (5), (15) e (16) tem-se:

$$
\frac{\gamma}{\zeta}=\frac{k}{m g}=-\frac{2 r_{r}}{r_{i}^{2}+r_{r}^{2}}
$$

Os coeficientes $\gamma, \zeta$, e a relação $\mathrm{k} / \mathrm{mg}$ podem ser determinados com as equações (15), (16) e (17) e consultados na Tabela 3 .
Na Figura 7, é apresentada a comparação entre os dados experimentais (curva azul), a solução teórica ajustada (curva verde) e a relação composta entre $\mathrm{R}(\theta(\mathrm{t}))$ para $\theta$ experimentais (curva vermelha) baseada na relação entre a distância do ponto de luz até o eixo de rotação do pêndulo $(\mathrm{R})$ e o tempo decorrido (t). Há uma correspondência boa entre as curvas verde e vermelha, porém uma discrepância entre estas duas com relação à azul.

É puramente geométrica a relação entre $\mathrm{R}$ e $\theta$, então não deveriam ocorrer divergências tão consideráveis entre as curvas vermelha e azul plotadas na Figura 7, pois uma difere da outra simplesmente pelo fato de que em uma $\mathrm{R}$ é calculado a partir da geometria e na outra $\mathrm{R}$ é obtido diretamente da filmagem que foi usada no Tracker®. A fim de entender essa discrepância, um gráfico que compara os dados experimentais (curva azul) e a solução teórica ajustada (curva verde) baseada na relação entre a distância do ponto de luz até o eixo de rotação do pêndulo $(\mathrm{R})$ e o ângulo de abertura do pêndulo $(\theta)$ é apresentado na Figura 8.

Uma possível explicação para o efeito consiste no fato de que as distâncias analisadas nas filmagens poderiam estar distorcidas com relação às distâncias reais. Um esquema do fenômeno pode ser visualizado na Figura 9.

A distorção exemplificada na Figura 9 teria se intensificado pela dimensão maiores do experimento e a perspectiva perdida no momento que a imagem é capturada. Essa distorção se intensifica quanto mais distante do centro da tela da filmagem o objeto se estende, como pode ser visualizado nos gráficos, onde a discrepância se intensifica nos momentos em que $\mathrm{R}$ atinge seus valores críticos (máximos e mínimos). Nota-se pela Figura 8 que para a Configuração 1 não conseguimos um casamento de dados consideravelmente próximos.

\subsection{Configuração Experimental 2}

Os dados recolhidos acerca da Configuração Experimental 2 podem ser consultados nas Figuras $(10), \sqrt{11}$ e $(12)$. Na Figura 10 observa-se que há uma correspondência muito satisfatória entre os dados experimentais e a curva ajustada em termos de $\theta(\mathrm{t})$. A curva resultante do ajuste de curvas possui os coeficientes explicitados na tabela (4).

Os coeficientes $\gamma, \zeta$, e a relação $\mathrm{k} / \mathrm{mg}$ podem ser determinados com as equações (15), (16) e (17) e consultados na tabela (5).

Na Figura 11, é apresentada a comparação entre os dados experimentais (curva azul), a solução teórica ajus-

Tabela 4: Coeficientes da equação (14) que define a curva verde explicitada na figura (10).

\begin{tabular}{llll}
\hline $\mathrm{c}_{1}\left(\mathrm{rad}^{-1}\right)$ & $\mathrm{c}_{2}\left(\mathrm{rad}^{-1}\right)$ & $\mathrm{r}_{\mathrm{i}}\left(\mathrm{s}^{-1}\right)$ & $\mathrm{r}_{\mathrm{r}}\left(\mathrm{s}^{-1}\right)$ \\
\hline$-0,04889$ & $-0,20126$ & $-0,3711$ & 9,01203 \\
\hline
\end{tabular}



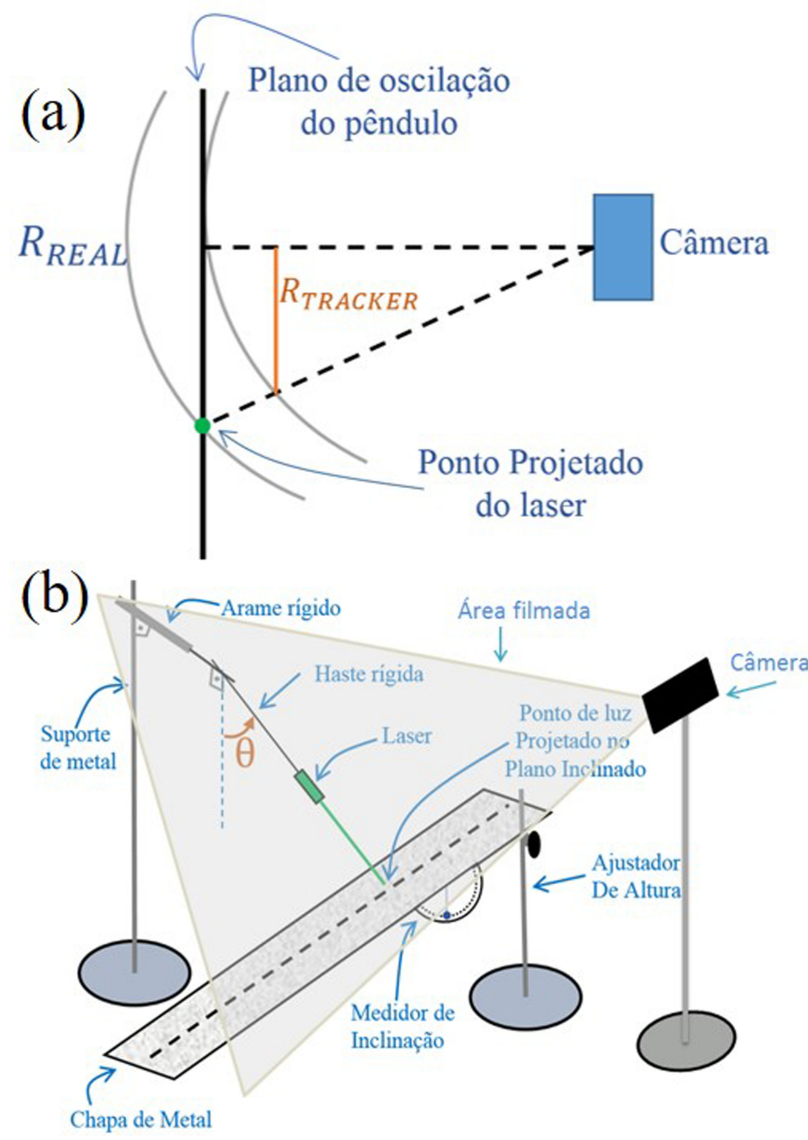

Figura 9: A) Esquema da captura de uma imagem a longa distância pela câmera, com enfoque na deformidade entre a distância $\mathrm{R}_{\mathrm{REAL}}$ e a distância $\mathrm{R}$ computada pelo Tracker ${ }^{\circledR}$. B) Posição da câmera com a área de captura.

Tabela 5: Coeficientes $\gamma, \zeta$, e a relação $\mathrm{k} / \mathrm{mg}$ para o experimento com plano inclinado em $10^{\circ}$ e com amplitude grande de $\theta$.

\begin{tabular}{lll}
\hline$\gamma\left[\left(\mathrm{s}^{-1}\right)^{-1}\right]$ & $\zeta\left[\left(\mathrm{s}^{-2}\right)^{-1}\right]$ & $\mathrm{k} / \mathrm{mg}\left[\mathrm{s}^{-1}\right]$ \\
\hline 0,097786 & 81,219 & 0,0012040
\end{tabular}

tada (curva verde) e a relação composta entre $\mathrm{R}(\theta(\mathrm{t}))$ para $\theta$ experimentais (curva vermelha) baseada na relação entre a distância do ponto de luz até o eixo de rotação do pêndulo $(\mathrm{R})$ e o tempo decorrido $(\mathrm{t})$ sobre o plano inclinado. Observamos que há uma superposição entre as curvas verde, vermelha e azul, porém ainda com discrepância acentuada nos pontos críticos da função, assim como foi observado na Figura 7.

Observando a Figura 12, notamos que essa configuração angular proporcionou um ajuste de $\mathrm{R} \times \theta$ muito melhor em relação à configuração anterior, apresentada na Figura 8, que possuía $\alpha$ pequeno.

\subsection{Configuração Experimental 3}

Na configuração angular 3, o ângulo de inclinação do plano, o $\alpha$, é alterado para $20^{\circ}$ e usamos uma abertura

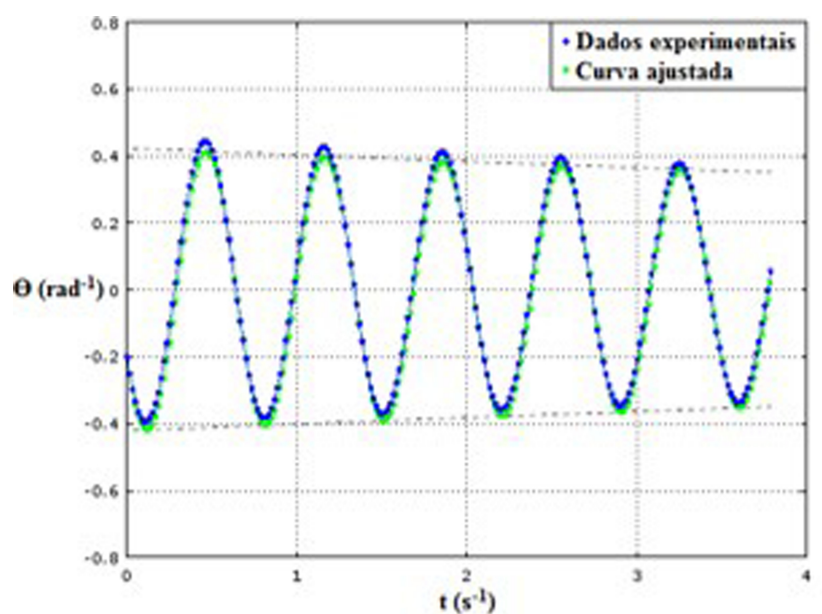

Figura 10: Gráfico com a comparação entre os dados experimentais (curva azul) e a solução teórica ajustada (curva verde) baseada na relação entre o ângulo de abertura do pêndulo $(\theta)$ e o tempo decorrido $(\mathrm{t})$.

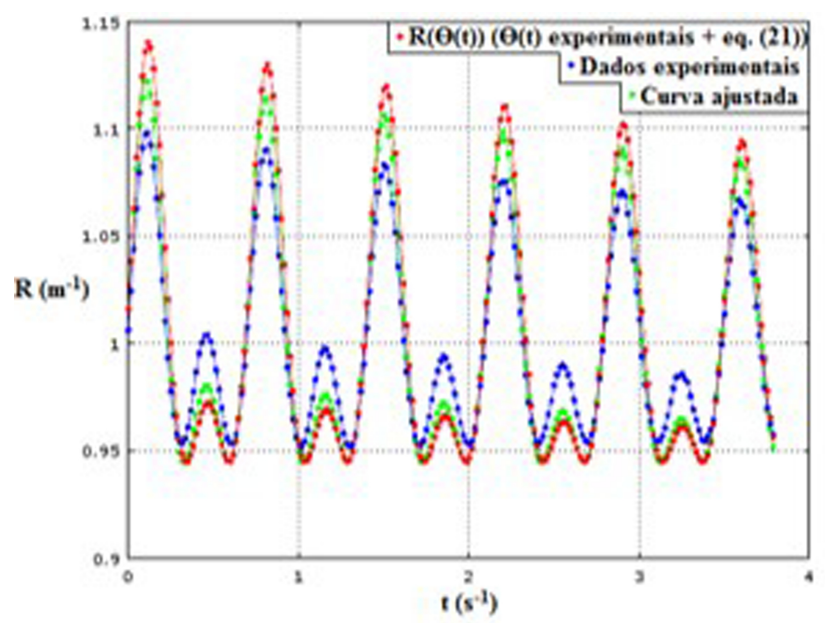

Figura 11: Gráfico com a comparação entre os dados experimentais, a solução teórica ajustada e a relação composta entre $\mathrm{R}(\theta(\mathrm{t}))$ para $\theta$ experimentais (curva vermelha) para configuração experimental 2 .

Tabela 6: Coeficientes da equação 14 que define a curva verde explicitada na figura (13).

\begin{tabular}{llll}
\hline $\mathrm{c}_{1}\left(\mathrm{rad}^{-1}\right)$ & $\mathrm{c}_{2}\left(\mathrm{rad}^{-1}\right)$ & $\mathrm{r}_{\mathrm{i}}\left(\mathrm{s}^{-1}\right)$ & $\mathrm{r}_{\mathrm{r}}\left(\mathrm{s}^{-1}\right)$ \\
\hline$-0,11427$ & 0,00196 & 0,13181 & 9,08691 \\
\hline
\end{tabular}

do pêndulo $(\theta)$ de ângulos pequenos $\left(<15^{\circ}\right)$. Os dados recolhidos para essa configuração podem ser consultados nas Figuras 13, 14 e 15.

Para esta configuração, nota-se que há uma correspondência muito satisfatória entre os dados experimentais e a curva ajustada em termos de $\theta(\mathrm{t})$, como apresentado na Figura 13. A curva resultante do ajuste de curvas possui os coeficientes explicitados na Tabela 6 . 


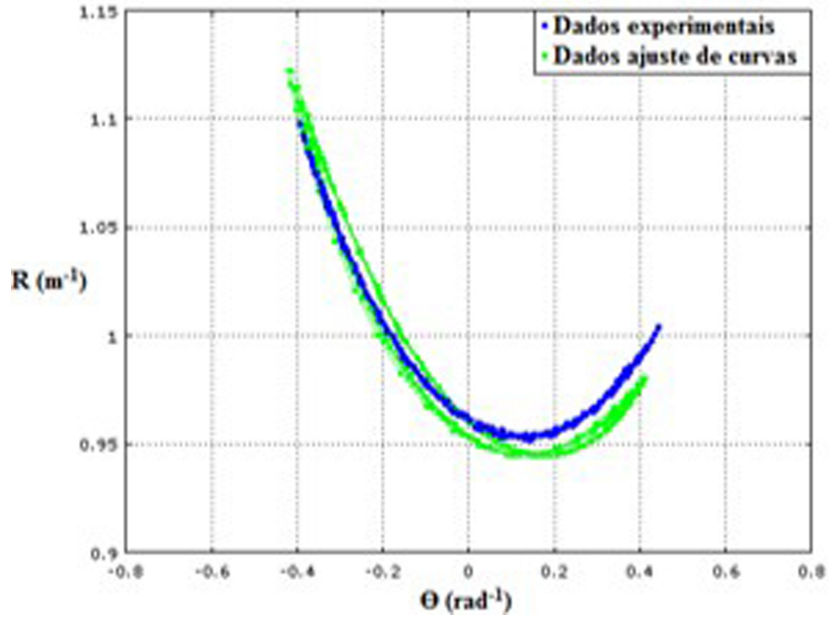

Figura 12: Gráfico com a comparação entre os dados experimentais (curva azul) e a solução teórica ajustada (curva verde) baseada na relação entre a distância do ponto de luz até o eixo de rotação do pêndulo $(\mathrm{R})$ e o ângulo de abertura do pêndulo $(\theta)$.

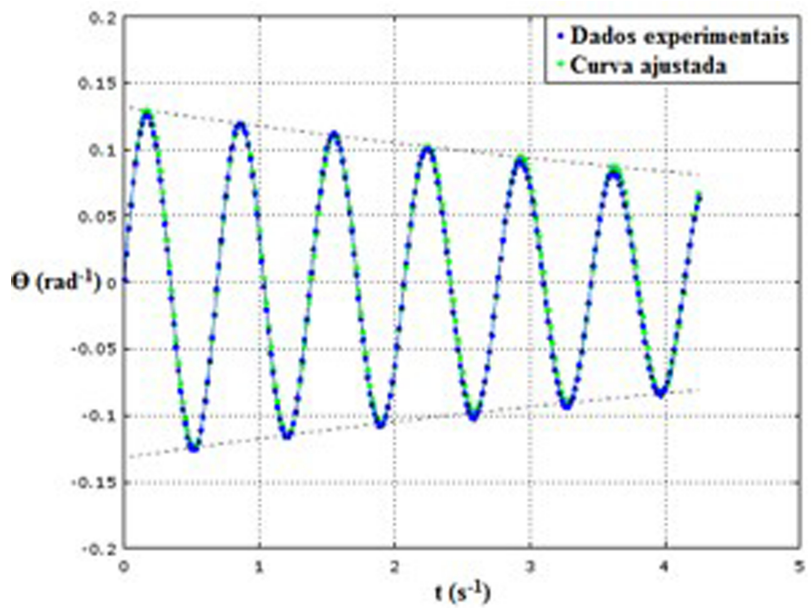

Figura 13: Gráfico com a comparação entre os dados experimentais (curva azul) e a solução teórica ajustada (curva verde) baseada na relação entre o ângulo de abertura do pêndulo $(\theta)$ e o tempo decorrido $(\mathrm{t})$.

Tabela 7: Coeficientes $\gamma, \zeta$, e a relação $\mathrm{k} / \mathrm{mg}$ para o experimento com plano inclinado em $20^{\circ}$ e com amplitude pequena de $\theta$.

\begin{tabular}{lll}
\hline$\gamma\left[\left(\mathrm{s}^{-1}\right)^{-1}\right]$ & $\zeta\left[\left(\mathrm{s}^{-2}\right)^{-1}\right]$ & $\mathrm{k} / \mathrm{mg}\left[\mathrm{s}^{-1}\right]$ \\
\hline 0,22854 & 82,585 & 0,0027674 \\
\hline
\end{tabular}

Os coeficientes $\gamma, \zeta$, e a relação $\mathrm{k} / \mathrm{mg}$ podem ser determinados com as equações (15), (16) e (17) e consultados na tabela (7).

A figura 14 apresenta a comparação entre os dados experimentais, a solução teórica ajustada e a relação composta entre $\mathrm{R}(\theta(\mathrm{t}))$ para $\theta$ experimentais baseada na relação entre a distância do ponto de luz até o eixo de rotação do pêndulo ( $\mathrm{R}$ ) e o tempo decorrido (t) sobre a

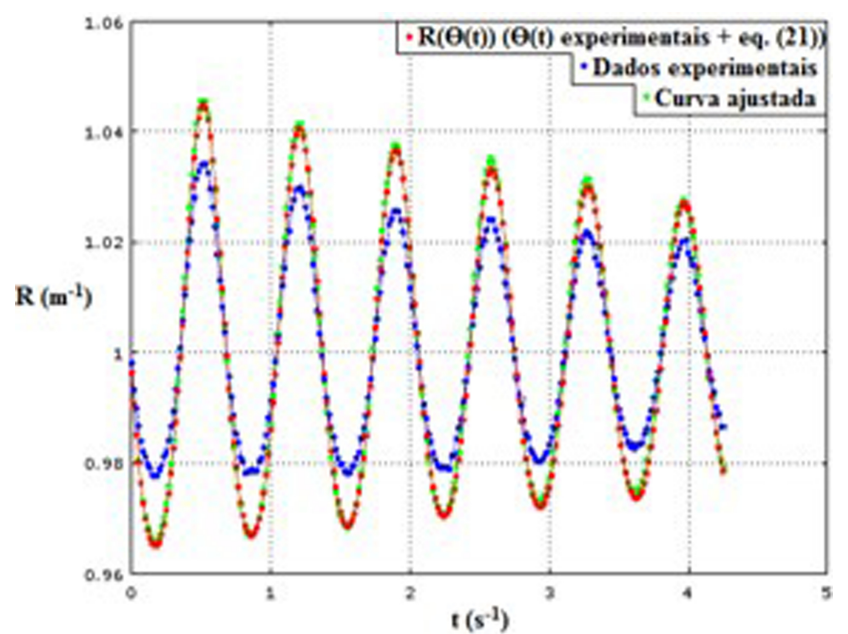

Figura 14: Gráfico com a comparação entre os dados experimentais (curva azul), a solução teórica ajustada (curva verde) e a relação composta entre $\mathrm{R}(\theta(\mathrm{t}))$ para $\theta$ experimentais (curva vermelha) baseada na relação entre a distância do ponto de luz até o eixo de rotação do pêndulo $(\mathrm{R})$ e o tempo decorrido $(\mathrm{t})$ sobre o plano inclinado em $20^{\circ}$ e com amplitude pequena de $\theta$.

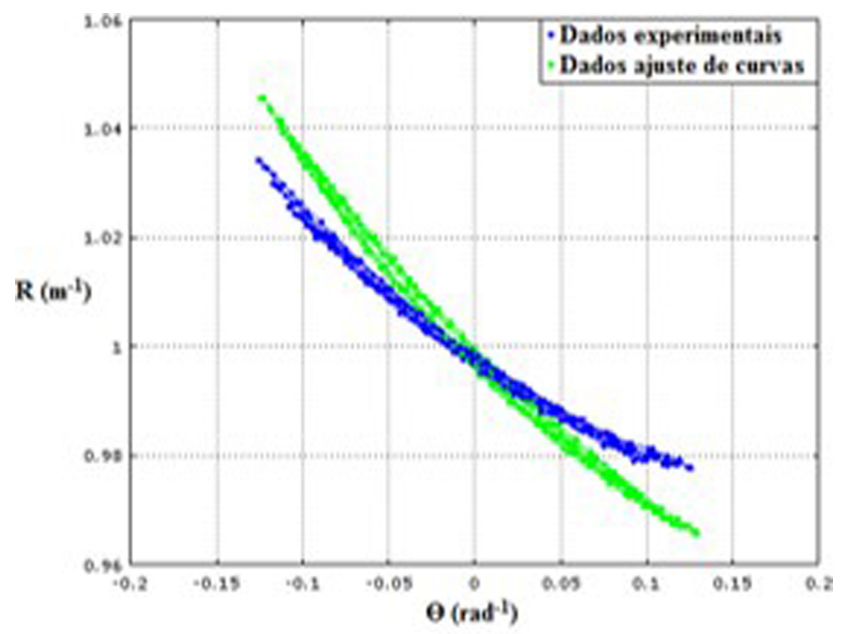

Figura 15: Gráfico com a comparação entre os dados experimentais (curva azul) e a solução teórica ajustada (curva verde) baseada na relação entre a distância do ponto de luz até o eixo de rotação do pêndulo $(R)$ e o ângulo de abertura do pêndulo $(\theta)$ sobre o plano inclinado em $20^{\circ}$ e com amplitude pequena de $\theta$.

configuração 3. Observamos que há uma superposição entre as curvas ajustadas e a relação composta entre $\mathrm{R}(\theta(\mathrm{t}))$ para $\theta$ experimentais, porém discrepância considerável entre estas duas com relação aos dados experimentais. $\mathrm{O}$ mesmo efeito já tinha sido observado nas outras configurações, veja Figuras 7 e 11.

Na Figura 15 apresentamos a relação entre o $\mathrm{R} \times \theta$. A alteração do ângulo $\alpha$ ocasionou uma difrença significativa no casamento desses dados. Observe que essa relação para a configuração 1 que possuía $\alpha$ pequeno na Figura 8. A mudaça para $\alpha$ grande na montagem proporcionou 
um casamento de dados um pouco melhor em relação à configuração anterior que utilizava o mesmo valor de $\theta$.

\subsection{Configuração experimental 4}

Por fim, na configuração experimental 4 a montagem adotou $\alpha=2^{\circ}$ e $\theta>15^{\circ}$, os dados recolhidos acerca dessa oscilação podem ser consultados nas Figuras 16, 17 e 18.

Na Figura 16 nota-se que há uma correspondência muito satisfatória entre os dados experimentais e a curva ajustada em termos de $\theta(\mathrm{t})$. A curva resultante do ajuste de curvas possui os coeficientes explicitados na Tabela 8.

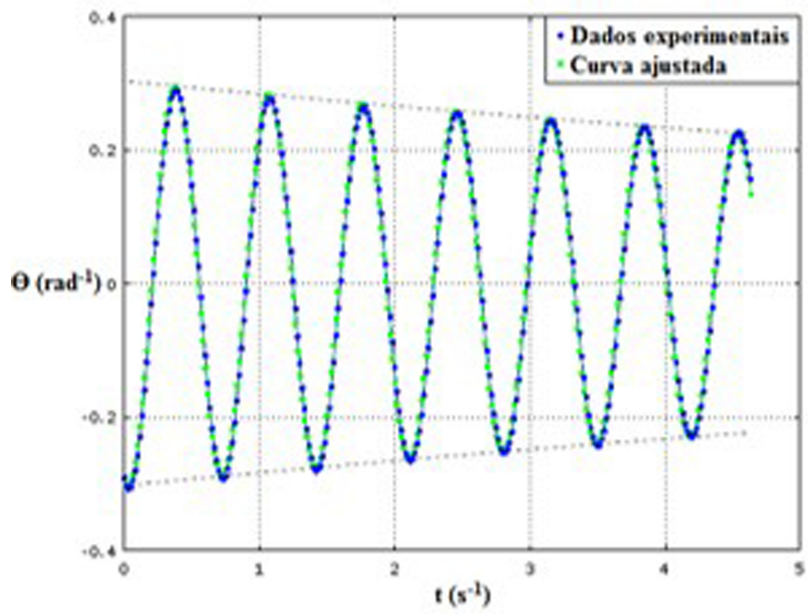

Figura 16: Gráfico com a comparação entre os dados experimentais (curva azul) e a solução teórica ajustada (curva verde) baseada na relação entre o ângulo de abertura do pêndulo $(\theta)$ e o tempo decorrido $(\mathrm{t})$ sobre o plano inclinado em $20^{\circ}$ e com amplitude grande de $\theta$.

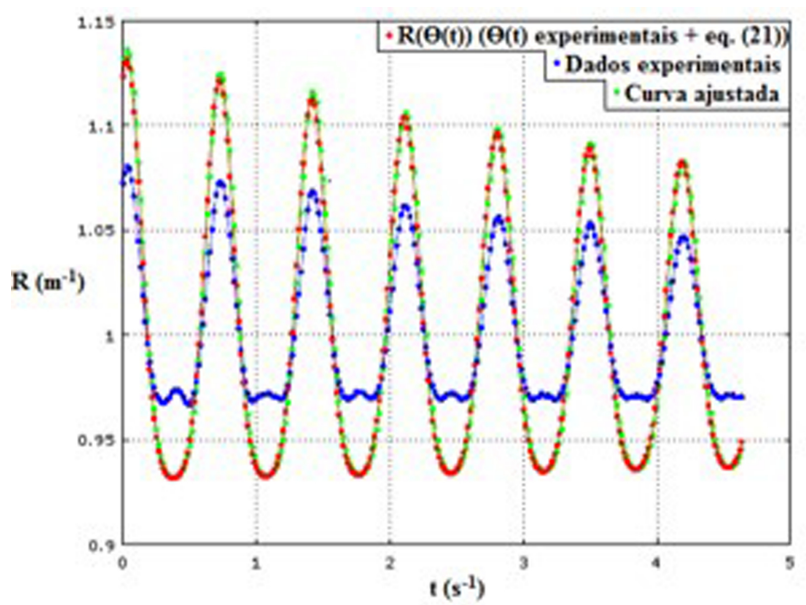

Figura 17: Gráfico com a comparação entre os dados experimentais (curva azul), a solução teórica ajustada (curva verde) e a relação composta entre $\mathrm{R}(\theta(\mathrm{t}))$ para $\theta$ experimentais (curva vermelha) baseada na relação entre a distância do ponto de luz até o eixo de rotação do pêndulo $(\mathrm{R})$ e o tempo decorrido $(\mathrm{t})$ sobre o plano inclinado.

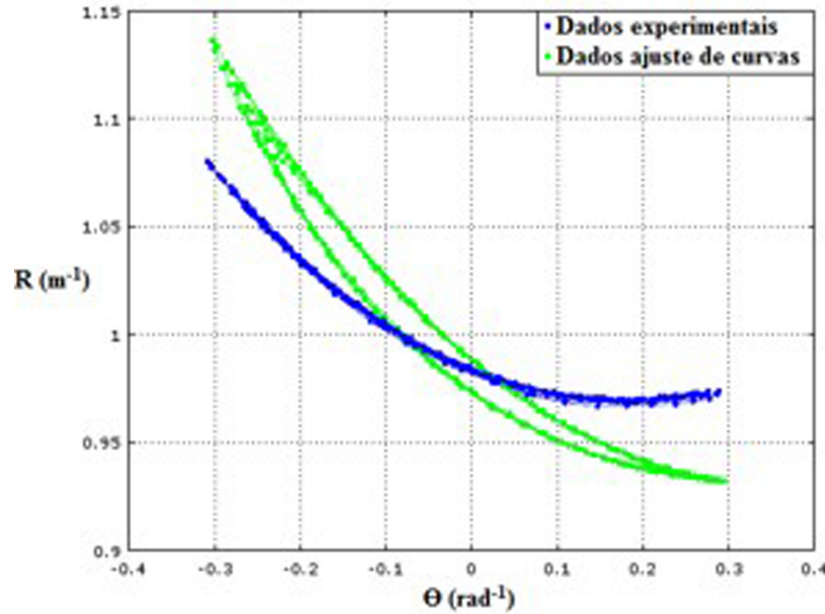

Figura 18: Gráfico com a comparação entre os dados experimentais (curva azul) e a solução teórica ajustada (curva verde) baseada na relação entre a distância do ponto de luz até o eixo de rotação do pêndulo $(R)$ e o ângulo de abertura do pêndulo $(\theta)$ sobre o plano inclinado em $20^{\circ}$ e com amplitude grande de $\theta$.

Os coeficientes $\gamma, \zeta$, e a relação $\mathrm{k} / \mathrm{mg}$ podem ser determinados com as equações (15), (16) e (17) e consultados na tabela (9).

$\mathrm{Na}$ relação entre $\mathrm{R}$ e t, para a configuração experimental 4, apresentada na Figura 17, nota-se que há uma correspondência boa entre as curvas verde e vermelha, porém discrepância considerável entre estas duas com relação à azul, assim como foi encontrada em todas as configurações instrumentais (veja Figuras 7, 11 e 14).

Na comparação entre $\mathrm{R}$ e $\theta$, mostrada na Figura 18, vemos que a mudança para $\alpha$ grande na configuração da montagem proporcionou uma relação entre esses dados um pouco pior do que é apresentação para Configuração Experimental 2, que possuía $\alpha$ pequeno e a mesma abertura $\operatorname{em} \theta$.

\subsection{Comparação}

Em síntese pode-se concluir que a melhor montagem experimental, em termos de obtenção de dados próximos do esperado teoricamente, consiste na montagem 3 , com $\alpha$ grande e $\theta$ pequeno. A razão $\mathrm{k} / \mathrm{mg}$ para todas as

Tabela 8: Coeficientes da equação 14 que define a curva verde explicitada na figura (16).

\begin{tabular}{llll}
\hline $\mathrm{c}_{1}\left(\mathrm{rad}^{-1}\right)$ & $\mathrm{c}_{2}\left(\mathrm{rad}^{-1}\right)$ & $\mathrm{r}_{\mathrm{i}}\left(\mathrm{s}^{-1}\right)$ & $\mathrm{r}_{\mathrm{r}}\left(\mathrm{s}^{-1}\right)$ \\
\hline$-0,06536$ & $-0,29114$ & $-0,0824$ & 9,06967 \\
\hline
\end{tabular}

Tabela 9: Coeficientes $\gamma, \zeta$, e a relação $\mathrm{k} / \mathrm{mg}$ para o experimento com plano inclinado em $20^{\circ}$ e com amplitude grande de $\theta$.

\begin{tabular}{lll}
\hline$\gamma\left[\left(\mathrm{s}^{-1}\right)^{-1}\right]$ & $\zeta\left[\left(\mathrm{s}^{-2}\right)^{-1}\right]$ & $\mathrm{k} / \mathrm{mg}\left[\mathrm{s}^{-1}\right]$ \\
\hline 0,13072 & 82,263 & 0,0015890
\end{tabular}


configurações anteriores podem ser consultados na Tabela 10 .

\section{Simulação}

Uma simulação do Pêndulo Simples com Laser no Plano Inclinado foi feita utilizando o software VPython com a versão 2.7.10 da linguagem Python. O material está disponível em www.fis.ita.br/phyton.

As variáveis referenciadas na simulação, apresentados na Figura 19, são as mesmas que estão presentes no artigo. Veja na Tabela 11.

Rodando a simulação, na janela "Controls"as variáveis podem ser ajustadas clicando e arrastando a barra deslizante. Na Tabela 12 são apresentados os intervalos de alcance de cada uma das variáveis da simulação.

A simulação pode ser interrompida clicando-se no botão "Parar"e reiniciada clicando-se no botão "Iniciar". O botão "Limpar"apaga os dados exibidos nos gráficos e deixa a simulação em pausa.

Os botões "Sub. Crítico", "Critico"e "Sup. Crítico"configuram as variáveis para as respectivas situações de amor-

Tabela 10: Valores da razão k/mg em função da configuração utilizada.

\begin{tabular}{ll}
\hline Configuração utilizada & $\mathrm{k} / \mathrm{mg}\left(\mathrm{s}^{-1}\right)$ \\
\hline$\alpha=1^{\circ}$ e $\theta<15^{\circ}$ & 0,0039891 \\
$\alpha=10^{\circ}$ e $\theta>15^{\circ}$ & 0,0012040 \\
$\alpha=2^{\circ}$ e $\theta<15^{\circ}$ & 0,0027674 \\
$\alpha=2^{\circ}$ e $\theta>15^{\circ}$ & 0,0015890 \\
\hline
\end{tabular}

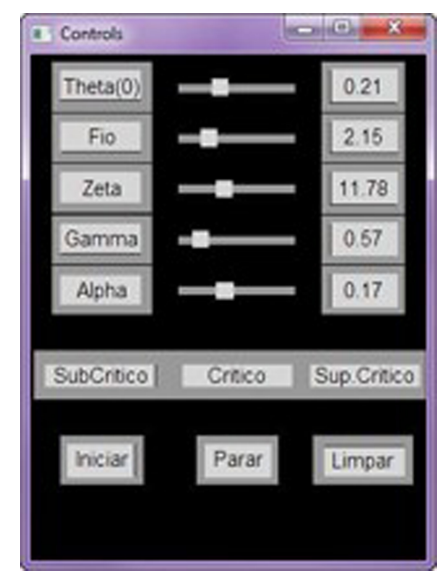

Figura 19: Painel que controla os parâmetros da simulação.

Tabela 11: Apresentação e Descrição das variáveis envolvidas no problema.

\begin{tabular}{ll}
\hline Variável & Descrição \\
\hline Fio & Comprimento do fio. \\
$\theta$ & Ângulo entre a vertical e o fio. \\
$\zeta$ & Quadrado da frequência angular natural. \\
$\gamma$ & Coeficiente de amortecimento. \\
$\alpha$ & Ângulo de inclinação do plano. \\
$\mathrm{R}$ & Distância entre o ponto onde está fixado o fio e a \\
& projeção da luz do laser no plano inclinado. \\
\hline
\end{tabular}

Tabela 12: Variáveis presentes na simulação com os intervalos que podem ser analisados.

\begin{tabular}{lll}
\hline Variável & Unidade & Alcances \\
\hline Fio & $\mathrm{Cm}$ & 0.5 a 7.8 \\
$\theta$ & $\mathrm{Rad}$ & 0.0 a 0.59 \\
$\zeta$ & $\mathrm{rad}^{2} / \mathrm{s}^{2}$ & 0.0 a 30.0 \\
$\gamma$ & $\mathrm{rad} / \mathrm{s}$ & 0.0 a 3.0 \\
$\alpha$ & $\mathrm{Rad}$ & $0.0 \mathrm{a} 0.44$ \\
\hline
\end{tabular}

tecimento Subcrítico, Crítico e Supercrítico e servem para exemplificar tais movimentos.

A Figura 20 mostra o gráfico de "Theta"em função do tempo "t"em vermelho.

O simulador possibilita, para fins didáticos, mostrar o comportamento do pêndulo com laser com alterações em suas variáveis.

Isto permite aos estudantes compreender a influência de cada um dos parâmetros apresentados na Tabela 12.

\section{Conclusão}

Esse trabalho só foi possível de ser realizado devido a nova proposta de ensino que estamos aplicando no laboratório experimental de física I para alunos que estão no $1^{\circ}$ ano de engenharia do ITA, isto é, juntar o uso da metodologia PBL com a liberdade dos estudantes proporem o experimento a serem desenvolvidos e analisados. Essa proposta permite entre outras coisas, que os estudantes desenvolvam muitas outras habilidades e competências do que no modo tradicional de ensino que é utilizado hoje, atendendo assim, as habilidades exigidas na Diretrizes Curriculares Nacionais do Curso de Graduação em Engenharia (2002), no que diz respeito competências e habilidades gerais das Engenharias, como (a) aplicar conhecimentos matemáticos, (b) projetar e conduzir experimentos e interpretar resultados, (c) resolver problemas de engenharia e (d) Desenvolver e/ou utilizar novas ferramentas e técnicas. Outra característica muito importante, é o desafio do novo e do desconhecido, isto é, poder pensar e resolver situações experimentais completamente novas e desafiadoras. Pelos resultados discutidos e apresentados

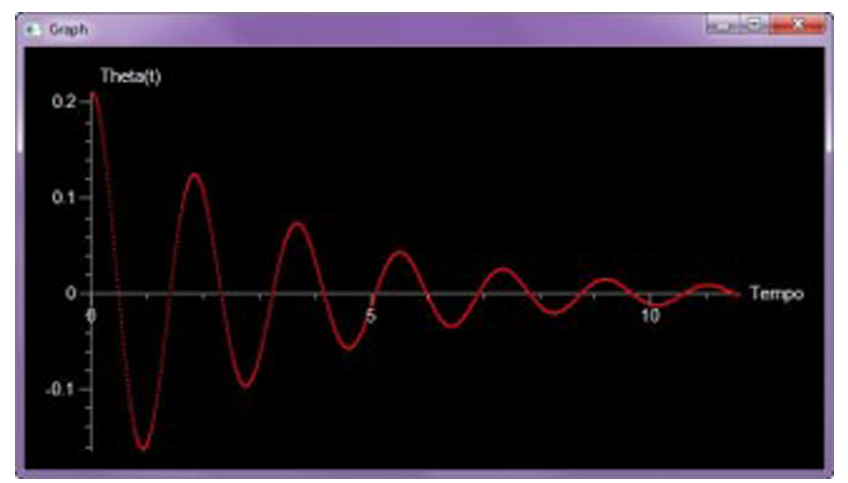

Figura 20: Um dos gráficos apresentados na simulação. 
anteriormente todas essas habilidades foram claramente trabalhadas nesse experimento.

Nota-se pelas Figuras 12 e 18 que a mudança para $\alpha$ grande na configuração da montagem proporcionou um casamento de dados pouco pior em relação à configuração anterior, que possuía $\alpha$ pequeno

Em síntese pode-se concluir que a melhor montagem experimental, em termos de obtenção de dados próximos do esperado teoricamente, consiste na montagem com $\alpha$ grande e $\theta$ pequeno. Entretanto, quanto à justificativa, não se pode afirmar nada veementemente, pois a questão da distorção na filmagem - devido às dimensões do experimento - pode ter tido forte influência no experimento e essa influência não pode ser determinada tão facilmente a não ser, é claro, num experimento especialmente focado nessa questão.

Ademais, destacamos que as figuras 10 e 16 apresentam ângulos $\theta$ maiores que $15^{\circ}$ porém próximo de $30^{\circ}$, tornando a relação $\operatorname{sen} \theta \approx \theta$ uma aproximação exagerada para a situação em questão. Isso implica em erros maiores que $5 \%$ ao não adotar a expansão de Taylor como solução.

A fim de se ter uma noção quantitativa da incerteza associada aos dados medidos pelo Tracker ${ }^{\circledR}$, foi realizada também uma filmagem com experimento em sua configuração parada. Essa configuração permitiu quantificar a influência dos ruídos nos dados que o Tracker®gerou. Abaixo seguem os gráficos de $\mathrm{R}(\mathrm{t}), \theta(\mathrm{t})$ e $\mathrm{R}(\theta)$ obtidas com o pêndulo parado, conforme figuras (21), (22) e (23) respectivamente.

A partir dos dados recolhidos e da fórmula de desvio padrão amostral pode-se calcular o desvio padrão amostral de $\mathrm{R}(\mathrm{t})$ e $\theta(\mathrm{t})$, os dados relacionados a esse cálculo podem ser consultados na tabela (13).

Dessa forma, realizando as aproximações, tem-se que:

$$
\mathrm{R}_{\text {PARADO }}=(1,00047 \pm 0,00003) \mathrm{m}
$$

\section{Perturbações R x t}

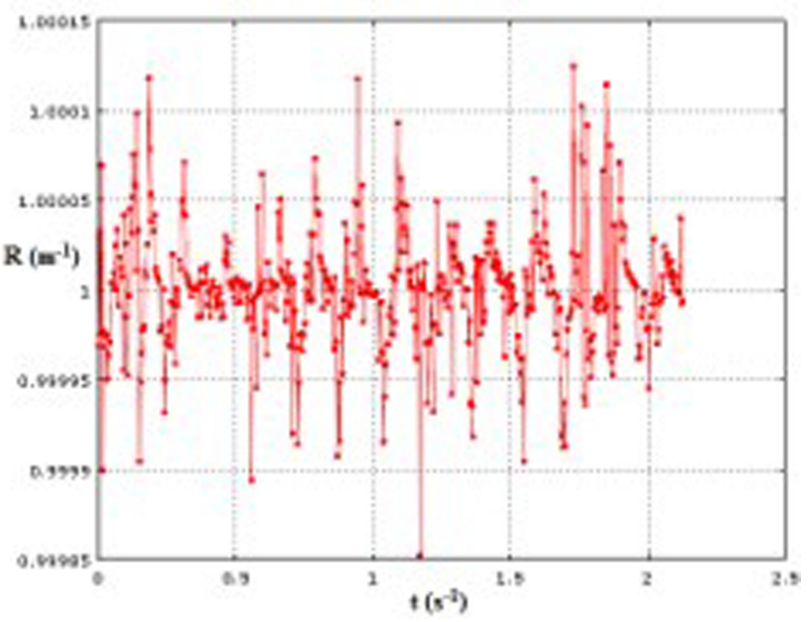

Figura 21: Gráfico com a relação entre $\mathrm{R}$ e $\mathrm{t}$ fornecida pelo Tracker ${ }^{\circ}$ para o experimento em sua configuração parada.

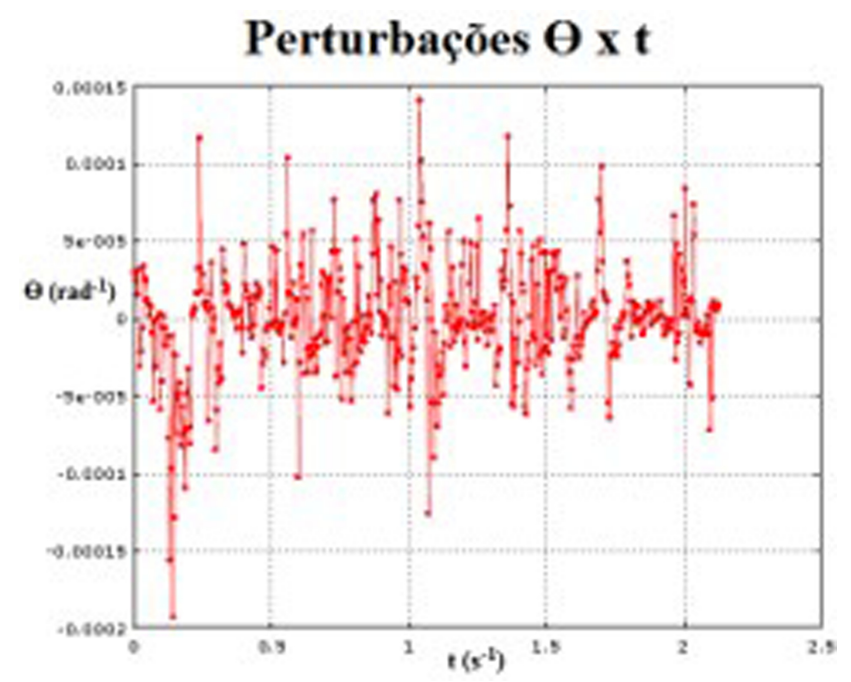

Figura 22: Gráfico com a relação entre $\theta$ e $\mathrm{t}$ fornecida pelo Tracker ®para o experimento em sua configuração parada.

\section{Perturbações R x $\boldsymbol{\theta}$}

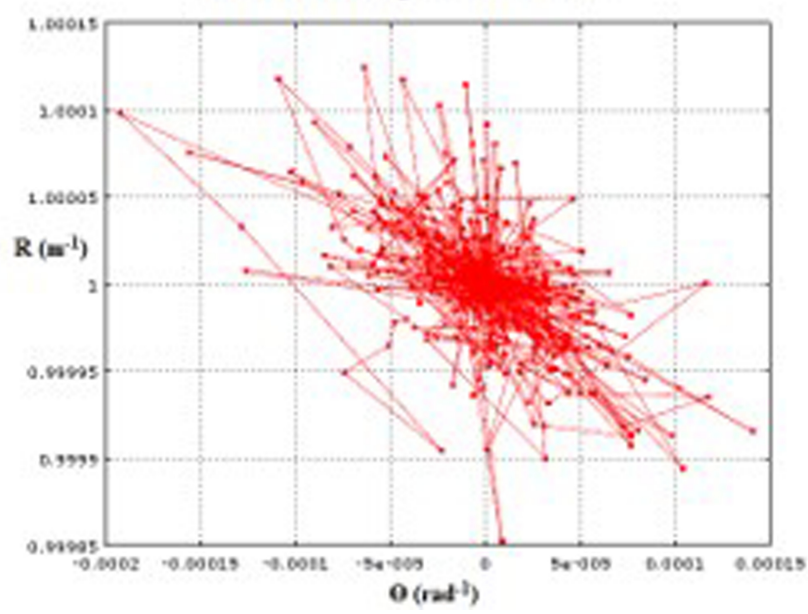

Figura 23: Gráfico com a relação entre $\mathrm{R}$ e $\theta$ fornecida pelo Tracker $\AA$ para o experimento em sua configuração parada.

$$
\theta_{\mathrm{PARADO}}=(0,0001 \pm 0,0002) \mathrm{rad}
$$

Nota-se que o desvio padrão para $\mathrm{R}$ é bem pequeno e não justifica a discrepância entre as curvas experimental e ajustada nos gráficos das figuras (12), (14) e (17), dessa forma, o motivo dessa discrepância não está relacionado à precisão da captura dos dados. Uma provável causa seria o fenômeno de distorção na filmagem mencionado anteriormente.

Tabela 13: Desvio padrão para $\mathrm{R}(\mathrm{t})$ e $\theta(\mathrm{t})$ fornecidos pelo Tracker ${ }^{\circledR}$ para o experimento em sua configuração parada.

\begin{tabular}{lll}
\hline \multicolumn{1}{c}{ Grandeza } & Média & Desvio Padrão \\
\hline $\mathrm{R}\left(\mathrm{m}^{-1}\right)$ & 1,000475 & $3,444408 \times 10^{-5}$ \\
$\theta\left(\mathrm{rad}^{-1}\right)$ & 0,000134 & 0,000220552 \\
\hline
\end{tabular}


Apontamos também que a equação (9) foi uma solução encontrada pelos estudantes, este sendo o principal motivo de manter a análise teórico-experimental com base nela, por mais que tornar-se-ia mais didático o uso da equação do oscilador harmônico amortecido na forma exponencial.

Por fim, para fins didáticos no qual esse experimento é proposto, os resultados experimentais obtidos estão dentro do esperado para um problema complexo como esse que foi analisado. As comparações entre os resultados experimentais e teóricos que foram apresentadas mostram que para algumas condições os resultados são aceitáveis. Para um maior aprofundamento das análises, devemos desenvolver modelos teóricos mais complexos que levem em consideração problemas de arrasto dos objetos bem como o formato dos mesmos. Outro resultado concreto foi o desenvolvimento de uma simulação em Python do experimento realizado, permitindo realizar simulações antes de realizar as atividades experimentais propriamente ditas.

\section{Agradecimentos}

Priscila Freitas-Lemes e Douglas Carlos Vilela agradecem a CAPES pelo apoio financeiro.

\section{Referências}

[1] D. C. Zanotta, E. Cappelletto, M. T. Matsuoka, Revista Brasileira de Ensino de Física 33, 2313 (2011).

[2] V. R. A. Pereira, C. R. M. Hayashi, R. F. Junior, Rev. Tecnol. Soc., 12, 111 (2016).

[3] M. S. T. Araújo, M. L. V. S. Abib, Revista Brasileira de Ensino de Física 25, 176 (2003).

[4] T. A. Borges, Cad. Bras. Ens. Fís., 19, 291 (2002).

[5] A.F. Zompero, C.E. Laburú, Rev. Ensaio, 13, 67 (2011).

[6] , H. Pekelman, A. Mello-Jr, A importância dos laboratórios no ensino de engenharia mecânica, in Anais do XXXII Congresso Brasileiro de Ensino de EngenhariaCOBENGE, Brasília, 2004.

[7] L. Helle, P. Tynjälä, E. Olkinuora, Higher Education 51, 287 (2006).

[8] F. Bouquet, Project-based physics labs using low-cost open-source hardware (2016)

[9] H. Goldstein, C. P. Poole, J. L. Safko, Classical Mechanics (Addison-Wesley, 2001) $3^{\text {rd }}$ ed.

[10] GNU Octave. Disponível em http://www.gnu.org/ sof tware/octave/, acessado em 11/06/16.

[11] D. Brown, Tracker: Free Video Analysis and Modeling Tool for Physics Education, disponível em http://www. cabrillo.edu/ dbrown/tracker.

[12] W. Bonventi, Jr. N. Aranha, Revista Brasileira de Ensino de Física 37, 2504 (2015).

[13] L. Shao, J. Han, D. Xu, J. Shotton. IEEE Transactions On Cybernetics, 43 (2013). 